\title{
The ambiguous pruritogenic role of interleukin-31 in cutaneous T-cell lymphomas in comparison to atopic dermatitis: a review
}

\author{
Berenika Olszewska ${ }^{1}$, Małgorzata Sokołowska-Wojdyło', Joanna Lakomy², Roman J. Nowicki ${ }^{1}$
}

'Department of Dermatology, Venereology and Allergology, Medical University of Gdansk, Gdansk, Poland ${ }^{2}$ Department of Pathology, Medical University of Gdansk, Gdansk, Poland

Adv Dermatol Allergol 2020; XXXVII (3): 319-325

DOI: https://doi.org/10.5114/ada.2020.96260

\begin{abstract}
Cutaneous T-cell lymphomas (CTCLs) comprise a group of chronic heterogeneous diseases of unknown pathogenesis, characterized by non-specific skin lesions such as patches, plaques and tumours. CTCL is accompanied by persistent pruritus poorly responding to antihistamines and therefore significantly reducing quality of life in patients with lymphomas. According to research data, interleukin-31 (IL-31) contributes to initiation and maintenance of the inflammatory process of the skin and pruritus in inflammatory dermatoses such as atopic dermatitis (AD), which is well established. The studies of a similar role of IL-31 in CTCLs are less homogenous. Due to contradictory reports concerning IL-31 and CTCL we have analysed available literature to summarize its role, focusing on CTCL and AD.
\end{abstract}

Key words: mycosis fungoides, cutaneous lymphomas, pruritus, interleukin-31.

\section{Introduction}

Cutaneous T-Cell Lymphomas (CTCLS) represent a group of extra nodal non-Hodgkin lymphomas, which arise from malignant clonal transformation of T-cells in the skin. While CTCL primarily affects the skin, it may subsequently involve blood, lymph nodes and visceral organs. The most common subtype of CTCL is mycosis fungoides (MF), which according to WHO-EORTC classification accounts for approximately $60 \%$ of cases [1]. The symptoms of MF include patches, infiltrating plaques which can progress to tumours as well as erythroderma. Lymph nodes, blood and visceral involvement may follow the skin lesions in the minority of cases [1]. Sezary syndrome (SS) is an aggressive form of CTCL, comprising approximately $5 \%$ of CTCL cases [2]. It is defined by a triad: erythroderma, lymphadenopathy and clonal neoplastic proliferation of T-cells in the blood [1, 2]. Treatment of early-stage CTCL includes skin-directed therapies such as topical steroids, topical cytostatic agents and phototherapy [3, 4]. The advanced stage of CTCL is treated with systemic therapies such as interferon $\alpha$, bexarotene, chemotherapeutic agents, extracorporeal photopheresis, allogeneic hematopoietic stem cell transplantation (allo-HSCT) and others [3, 4]. CTCL is accompanied by persistent pruritus that affects between $60 \%$ and $90 \%$ of patients $[5,6]$. It is difficult to treat as antihistamines are usually ineffective and thus pruritus significantly reduces quality of life. Therefore, it seems that a nonhistaminergic pathway is involved in pathogenesis of CTCL's pruritus.

Four-helix bundle cytokine, interleukin-31 (IL-31), is a non-histamine pruritogen that is proven to be correlated with pruritus in atopic dermatitis [7, 8]. It belongs to the IL-6 cytokine family that includes IL-6, IL-11, IL-27, oncostatin M (OSM), ciliary neurotrophic factor (CNTF), leukaemia inhibitory factor (LIF), cardiotrophin-1 (CT-1) and cardiotrophin-like cytokine (CLC), all of which share glycoprotein 130 (gp130) as a receptor subunit [9-11]. In contrast, IL-31 uses IL-31 receptor $\alpha$ (IL-31 RA). Hence, IL-31 signals through a heterodimeric receptor consisting of IL-31 RA and oncostatin M receptor $\beta$ (OSMR) [9-11]. Through that receptor complex, IL-31 induces the activation of Janus kinase/signal transducer and activator of transcription (JAK/STAT), mitogen-activated protein kinase (MAPK) and phosphoinositide 3-kinase/AKT (PI3K/AKT) signalling pathway, which results in translocation to nucleus and then gene transcription [10, 11]. IL-31 is produced by a variety of cells, predominantly by

Address for correspondence: Berenika Olszewska MD, Department of Dermatology, Venereology and Allergology, Medical University of Gdansk, 1a Kliniczna St, 80-402 Gdansk, Poland, phone: +48 58349 25 80, e-mail: berenika.olszewska@o2.pl Received: 10.01.2019, accepted: 27.03.2019. 
CLA+ CD45RO+ memory T cells and also mast cells, monocytes/macrophages, dendritic cells [9-12]. T helper 2 cells (Th2), a subtype of T-cells, were demonstrated to be the primary source of IL-31 in human skin diseases [12-14]. The predominant tissues expressing IL-31 mRNA are skin, lung, nervous system and intestine ones [9-11]. IL-31 mRNA was also found in the testis, bone marrow, skeletal muscle, kidney, thymus, colon, trachea and dorsal root ganglia (DRG) $[9,10,13]$. IL-31 RA and OSMR are co-expressed on keratinocytes, activated monocytes and DRG $[9,10,13,14]$. However, constitutive expression of both receptor units is found in many other tissues such as thymus, testis, ovary, prostate, placenta, spleen, trachea, bone marrow ones suggesting that IL-31 may have potential pleiotropic physiological functions $[9,10]$.

According to recent research results, interleukin-31 (IL-31) is involved in the inflammatory process and initiation of pruritus in pruritic skin dermatoses indicating that a blockade of the IL-31 pathway may be an alternative to antihistamines in itch treatment. Moreover, recently, an anti-IL-31 receptor antibody has been shown to significantly improve pruritus in patients with atopic dermatitis. As CTCL and AD share some similarities, IL-31 has been suspected to play an analogous pruritogenic role in CTCL. However, the quantity of research on IL-31 role in CTCL is rather limited and conclusions are not homogeneous. CTCL shares many clinical, histological and immunological characteristics with AD. Both diseases show infiltration of the skin by skin homing T cells, predominance of Th2 immune response and pruritus [15-17]. Due to the fact that pruritogenic function of IL-31 in atopic dermatitis is well established, its role in $C T C L$ remains a promising target of investigation. In our work we have described the IL-31 role in AD and focused on publications concerning the pruritogenic role of IL-31 in CTCL and its potential association with the stage of the disease.

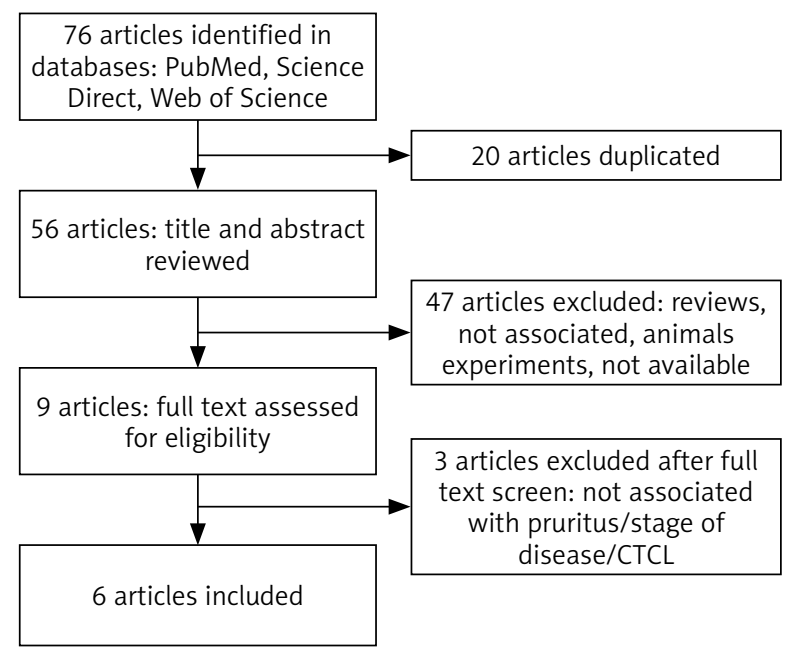

Figure 1. Flow diagram of the study according to PRISMA [20]

\section{Aim}

As there are several excellent systematic reviews comprehensively dealing with IL-31 role in $\operatorname{AD}[18,19]$, we conducted a systematic review of only $\mathrm{CTCL}$.

\section{Material and methods}

An extensive search in the literature was performed by two investigators for studies that approached the association between IL-31, pruritus and CTCL in humans. We performed a comprehensive literature review in line with the criteria published in the Preferred Reporting Items for Systematic Reviews and Meta-Analyses (PRISMA) guidelines. PubMed, Science Direct and Web of Science were the medical and scientific databases used in the literature retrieval. We used the following combination of searched key words: "interleukin-31", "cutaneous T-cell lymphomas", "mycosis fungoides", and "Sezary syndrome". Inclusion criteria: studies included IL-31 correlation with pruritus and/or a disease stage of CTCL, case-control research, no duplicated data in the study. Articles concerning diseases other than CTCL, pertaining to signalling pathways, biochemical properties, treatment association with IL-31 levels, animal studies, reviews, case reports, and personal experience summaries, studies not meeting the inclusion criteria of this study or in a language other than English were excluded. Relevant articles were screened by title and abstract, then the full text was assessed for eligibility.

\section{Results}

The flow diagram of this study is presented in Figure 1 [20]. We identified 76 potential studies and publications, 20 were excluded for duplication, 47 were excluded after title and abstract screening, while 3 were excluded after full text screening. Furthermore, articles were excluded for diseases other than CTCL, lack of relevance to pruritus or stage of the disease, review articles or animal studies. Key studies and findings are highlighted in Table 1.

\section{Solid pruritogenic role of IL-31 in atopic dermatitis}

Recent studies have been continuously extending knowledge of the complex pathophysiology of pruritus involving complicated interactions not only between multiple skin cells and the afferent nerve fibres but also new pruritogens, such as IL-31 and non-histaminergic receptors (Figure 2) [21-25]. However, the mechanism of pruritus brought by those mediators is still not known. IL-31 is a pleiotropic inflammatory cytokine involved in regulation of the inflammation and inducing pruritus [9-11]. As IL-31 RA and OSMR, subunits of the IL-31 receptor, are expressed in dorsal root ganglia and epidermis, IL-31 is indicated to be a missing link between the neural and immune system in the induction of inflammatory 
Table 1. Summary of interleukin-31 studies in CTCL regarding the IL-31 serum level or expression in the skin

\begin{tabular}{|c|c|}
\hline Reference & Conclusions \\
\hline Ohmatsu (2012) [53] & $\begin{array}{l}\text { - IL-31 serum level (ELISA) is significantly higher in CTCL than the healthy control group (CG) } \\
\text { - IL-31 serum level is significantly higher in the tumour stage than CG and patch and plaque stage } \\
\text { - IL-31 serum level is significantly higher in stage IV than CG and stage I } \\
\text { - IL-31 serum level correlates with serum sIL-2R and LDH level } \\
\text { - Conclusion: IL-31 serum level reflects the severity of CTCL }\end{array}$ \\
\hline Nobbe (2012) [40] & $\begin{array}{l}\text { - Significantly higher expression of IL-31 in the infiltration (skin immunohistochemistry) of AD compared } \\
\text { with MF/SS/psoriasis/alopecia areata/prurigo nodularis/notalgia paresthetica } \\
\text { - No differences in IL-31 expression in the infiltration of AD compared with pruritus sine materia } \\
\text { - Increased IL-31 immunoreactivity only in AD compared with other itching and Th2-weighted diseases } \\
\text { (pruritus sine materia/psoriasis inversa/prurigo nodularis/notalgia paresthetica/SS/alopecia areata/MF) } \\
\text { - No differences in the expression of IL-31 RA and OSMR in the dermal infiltration of skin diseases } \\
\text { - Conclusion: IL-31 plays a unique role in AD; increased IL-31 expression is not a general feature of itching } \\
\text { skin diseases }\end{array}$ \\
\hline Singer (2013) [52] & $\begin{array}{l}\text { - IL-31 mRNA levels (qRT-PCR) significantly correlate with marked pruritus in CTCL } \\
\text { - Statistically significant difference in IL-31serum level (ELISA) between pruritic and non-pruritic CTCL } \\
\text { patients } \\
\text { - No correlation between IL-31 mRNA or protein and patient stage } \\
\text { - Clinical resolution of pruritus correlates with a declining IL-31 protein level } \\
\text { - Conclusion: IL-31 is a potential anti-itch therapy }\end{array}$ \\
\hline Mobs (2014) [54] & $\begin{array}{l}\text { - Lack of significant differences between IL-31 serum level (ELISA) in MF and SS } \\
\text { - Lack of significant differences between CTCL stages } \\
\text { - Not significant but considerably higher IL-31 serum levels in severely pruritic than non-pruritic CTCL } \\
\text { - Expression of IL-31 in low levels in cell lines but not in the tumour cells in SS } \\
\text { - Conclusion: IL-31 does not play a central role in MF/SS pathogenesis }\end{array}$ \\
\hline Malek (2015) [51] & $\begin{array}{l}\text { - IL-31 serum level is significantly higher in CTCL than CG } \\
\text { - No statistically significant differences between IL-31 serum level in pruritic and non-pruritic CTCL } \\
\text { patients } \\
\text { - No correlation between IL-31 serum level (ELISA) and severity of pruritus (pruritus correlated with serum } \\
\text { IL-31 concentration only in stage Ib) }\end{array}$ \\
\hline Nattkemper (2016) [50] & $\begin{array}{l}\text { - IL-31 epidermal levels (immunofluorescence) significantly correlates with itch intensity } \\
\text { - No correlation between IL-31 epidermal levels and disease stage } \\
\text { - IL-31 is significantly elevated in the epidermis and dermal infiltration } \\
\text { - IL-31 RA and OSMR is significantly elevated only in epidermis } \\
\text { - IL-31 RA and OSMR levels significantly correlates with pruritus but not with the CTCL stage } \\
\text { - Conclusion: IL-31 may have an indirect effect on sensory nerves through epidermal neoplastic T-cells and } \\
\text { keratinocytes to transmit itch; IL-31 does not play an essential role in CTCL pathogenesis }\end{array}$ \\
\hline
\end{tabular}

itch. Series of research on transgenic mice showed that overexpression of IL-31 resulted in atopic-like dermatitis $[13,26]$. Another evidence of the pruritogenic role of IL-31 is induction of pruritus after IL-31 cutaneous injection [13]. Dillon et al. demonstrated that cutaneous injection of IL-31 in mice elicited pruritus after a couple of days, moreover pruritus persisted for a few days after administration had stopped [13]. Both intradermal and intravenous administration of IL-31 showed delayed onset of itch and long-lasting scratching behaviour in mice [27, 28]. Similarly, IL-31 induces transient scratching behaviour in primates-cynomolgus monkeys [29]. When it comes to humans, Havro et al. study indicated that IL-31 does not induce an immediate itch after a skin challenge [30]. Interestingly IL-31 administration caused strong and long-lasting erythema in almost all AD patients and healthy subjects suggesting its role in promoting local inflammation [30].
Lack of induction of immediate itch after IL-31 administration in $A D$ patients or healthy controls suggests that IL-31 acts indirectly via keratinocytes, secondary mediators and probably then C-fibres activation rather than its own receptors on skin neuronal endings. A recent study by Cevikbas et al. demonstrated that some itch neurons co-expressed transient receptor potential cation channel vanilloid subtype-1 (TRPV-1), transient receptor potential cation channel ankyrin subtype-1 (TRPA-1) and IL-31RA as targets for IL-31 Th2 mediated itch [14]. Jianghui Meng et al. showed for the first time that IL-31 increased synthesis of brain-derived natriuretic peptide, an itch mediator, in skin and DRG in AD [31]. It indicates a link between IL-31 mediated pruritus and sensory neurons. Several excellent reviews have covered the mechanisms and involvement of the IL-31 and its receptor axis in pruritus and inflammation process [32-34]. 


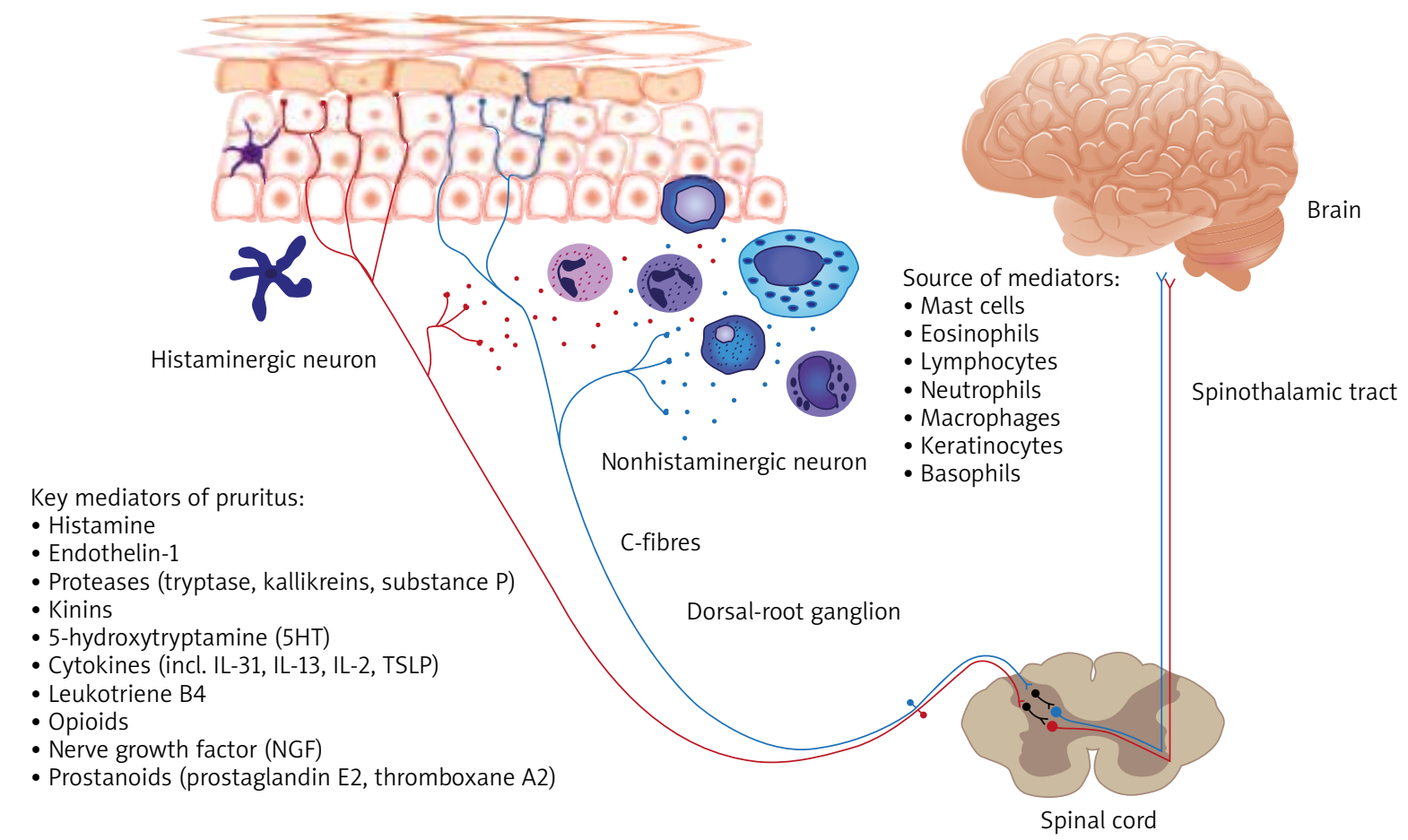

Figure 2. Schematic diagram of the pruritus pathway with main producers and itch mediators. The signal following the binding of itch mediators (pruritogens) to specific receptors (pruriceptors) from the skin is transmitted by itch-selective C-fibres (histamine-dependent itch fibres and histamine-independent itch fibres) to dorsal root ganglia and spinal neurons in lamina I of the dorsal horn, the itch signal ascends in the contralateral spinothalamic tract which projects to the thalamus. Then itch is transmitted to the somatosensory cortex, involved in recognition of itch

Kasutani et al. showed that intravenously administered IL-31 receptor antibody reduced itch in mice [28]. In a separate study by Grimstad et al., anti-IL-31 antibodies reduced itch in a murine model of atopic dermatitis [26]. In primates as in mice, the IL-31-induced scratching was suppressed by anti-human IL-31RA monoclonal antibody [29]. These studies elicited clinical trials (phase 1 and phase 2 clinical) in AD on human monoclonal interleukin-31 antagonist, nemolizumab (CIM331), which turned out to decrease pruritus [35]. The results have shown pruritus reduction up to $50 \%$ (visual analogue scale, VAS) after 4 weeks' use of antibodies [35]. Moreover, AD patients treated with CIM331 had improved sleep efficacy and required less topical hydrocortisone use [35]. A phase 2 trial comparing effects of nemolizumab with placebo found that the monthly administration improved pruritus among patients with severe atopic dermatitis [36]. It seems that anti-IL-31 antibody is likely to be efficient treatment for pruritic diseases, however its effectiveness in other diseases apart from AD needs further studies.

Research performed on transgenic mice has strongly suggested a potential role of IL-31 in AD and other inflammatory dermatoses with pruritus. Nowadays the prevailing view of the IL-31 role is pruritogenic cytokine.
The IL-31 level has been shown to be elevated in plasma/ serum in patients with $\operatorname{AD}[7,8,37,38]$. Moreover, IL-31 expression was increased in pruritic skin in $A D$ compared with healthy individuals [7, 12, 39, 40]. Not only lesional skin but also non-lesional skin of AD patients expressed increased IL-31 mRNA levels [7]. Sonkoly et al. and Raap et al. showed that IL-31 serum levels in AD correlated with disease activity and severity [7, 8]. Similarly, in another study, Kim et al. demonstrated a correlation between serum IL-31 levels and the subjective itch intensity in $A D$ patients [37]. Furthermore, serum IL-31 levels in AD correlated with cutaneous IL-31 mRNA, total serum IgE, eosinophil cationic protein and disease severity [33]. Contrary to these findings, Sokołowska-Wojdyło et al. reported no correlation between the IL-31 serum level and severity of pruritus in AD patients [38]. Neis et al. reported no correlation between the IL-31 mRNA level and both disease severity and IgE level in AD patients [39]. Nevertheless, a significantly higher expression of IL-31 in AD patients than in healthy individuals was observed in every single study suggesting its role in AD. Several studies showed an association of the IL-31 gene polymorphism with an increased risk of AD and non-atopic eczema [41, 42].

Moreover, several studies confirmed the role of IL-31 in $A D$ in paediatric population, demonstrating a correla- 
tion between the IL-31 level and severity of AD [43, 44]. On the other hand, Raap et al. and Siniewicz-Luzeńczyk et al. revealed no correlation between serum IL-31 and itch intensity $[43,45]$. Past reports strongly indicated IL-31 involvement in inducing both inflammation and pruritus in AD. Lack of correlation between IL-31 and pruritus might be explained by subjective evaluation of itch severity score. In conclusion, the serum IL-31 level is increased in AD compared to healthy subjects [37, 43-45]. The relationship between IL-31 and AD severity is less clear. In some studies [37, 43, 44, 46, 47], a positive correlation between IL-31 and AD severity was observed, while others showed no correlation [39, 45].

Observations of transgenic mice over-expressing IL-31, developing a skin phenotype closely mimicking atopic dermatitis [13] and increased levels of IL-31 in serum and skin of $A D$ patients elicited research on the IL-31 role in the inflammation process. AD is mediated by $T$ cells with a predominant Th2 type response and an increased number of cytokines IL-4, IL-5, IL-13 in acute skin lesions [48]. Interestingly, the IL-31 level correlates with the expression of IL-4 and IL-13. Thus, IL-31 was suggested to be involved in Th2-weighted inflammation diseases [14, 35, 49]. Nobbe et al. examined involvement of IL-31 in skin conditions according to the inflammatory profile and pruritus, namely T helper 1 cells (Th1) inflammation, Th2 inflammation and no inflammation [40]. Increased IL-31 immunoreactivity was observed only in AD compared with samples from other Th2-weighted diseases (MF, SS, alopecia areata), Th1-weighted diseases (psoriasis vulgaris, prurigo nodularis, psoriasis inversa) and conditions without inflammation (perilesional skin, pruritus sine materia, notalgia paresthetica) [40]. It was suggested that IL-31 is a mediator specific to atopic dermatitis rather than a pruritogen or general mediator in Th-weighted diseases. On the other hand, Sonkoly et al. revealed that lesional and non-lesional $A D$ as well as prurigo nodularis skin expressed increased IL-31 mRNA levels [7].

\section{IL-31 in the course of cutaneous T-cell lymphomas}

Published data suggest that IL-31 with its receptor components (OSMR and IL-31 RA) are involved in AD and there are strong conditions for its involvement in inducing both pruritus and Th2-mediated inflammation. Furthermore, CTCL mimics AD when it comes to clinical presentation or laboratory findings (e.g. eosinophilia, increased IgE levels). Given this strong association, IL-31 was also investigated in CTCL. Key studies and findings are summarized in Table 1 . There are only a few studies investigating the IL-31 role in CTCL due to the rarity of the disease. So far, only one study investigating IL-31 and its receptors expression in the CTCL skin was published [50]. Correlation of IL-31 levels in the skin and in blood of CTCL patients has not been studied yet. The studies were limited to investigation of IL-31 mRNA levels in peripheral blood mononuclear cells (PBMCS), levels of IL-31 in blood serum and the level of IL-31 and its receptors in the skin of CTCL patients and healthy individuals [50-54].

In addition to $A D, C T C L$ was reported to overexpress IL-31 in serum and in the skin. Alike AD, patients with CTCL exhibit increased levels of serum IL-31 compared to healthy controls [51-54]. Moreover, Ohmatsu et al. reported a correlation between serum IL-31 levels and advanced CTCL disease stage, thus IL-31 is suggested to play a role in CTCL pathogenesis [53]. Singer et al. confirmed that IL-31 mRNA is increased in CTCL and also showed a significant correlation between IL-31 and patients experiencing marked pruritus [52]. Moreover, they reported a correlation between a decreased IL-31 serum level and resolution of pruritus in CTCL patients. No correlation between IL-31 and stage of the disease was seen, probably due to the fact that majority of patients were in advanced stages [52]. This is consistent with Mobs et al. findings, confirming lack of interrelation between the IL-31 level and CTCL stage [54]. Overexpressed IL-31 was noted in CTCL and was suggested to be involved in CTCL's pruritus [52-54]. However, Malek et al. study did not confirm those findings, indicating that IL-31 is rather involved in CTCL pathogenesis [51]. There were no differences in the IL-31 level between pruritic and non-pruritic patients with CTCL, pruritus correlated with serum IL-31 concentration only in stage $\mathrm{lb}$ [51]. Malek et al. research results do not match earlier findings and need further studies. On the other hand, Nattkemper et al. examined expression of IL-31 and its receptors (OSMR and IL-31RA) in the skin in CTCL patients [50]. IL-31 was significantly elevated in the epidermis and dermal infiltration, OSMR and IL-31RA were significantly elevated only in epidermis. Like in most of the previous research results, IL-31 level correlated with itch intensity [52, 54]. Similar to Singer et al. study results, the number of patients in early and advanced stages was rather small $[50,52]$. It might be a possible reason for lack of correlation between IL-31 expression and disease stage. Nattkemper et al. confirmed these suggestions, indicating that IL-31 is involved in pruritus pathogenesis but it is irrelevant to pathogenesis of the disease itself [50].

\section{Conclusions}

Not only pathogenesis of CTCL but also pathophysiology of pruritus in CTCL remain unclear. It is likely that unknown proinflammatory agents produced in the microenvironment of the skin are involved, both in tumour growth and induction of pruritus in CTCL. The data presented in publications mentioned above have been the reason for continuous studies concerning the role of IL-31 in CTCL and pruritus. We already know that expression of IL-31 in serum in pruritic dermatoses including CTCL, according to some authors, is elevated. However, the question is whether overexpression of IL-31 in serum is 
associated with pruritus, disease itself or both. We also do not know whether IL-31 is directly associated with development of skin lesions in CTCL. Due to the great number of studies, IL-31 is best known for its pruritogenic role in $A D$, in contrast to its role in CTCL. IL-31 seems to play an important role in the signalling pathway involved in pruritus, moreover it is suggested to play a proinflammatory and immunomodulatory role. Undoubtedly, IL-31 is a promising cytokine, however its role in CTCL is not fully understood and it needs further studies.

\section{Conflict of interest}

The authors declare no conflict of interest.

\section{References}

1. Willemze R, Jaffe ES, Burg G, et al. WHO/EORTC classification for cutaneous lymphomas. Blood 2015; 105: 3768-85.

2. Scarisbrick JJ, Kim YH, Whittaker SJ, et al. Prognostic factors, prognostic indices and staging in mycosis fungoides and Sézary syndrome: where are we now? Br J Dermatol 2014; 170: 1226-36.

3. Trautinger F, Knobler R, Willemze R, et al. EORTC consensus recommendations for the treatment of mycosis fungoides/ Sézary syndrome. Eur J Cancer 2006; 42: 1014-30.

4. Wilcox RA. Cutaneous T-cell lymphoma: 2011 update on diagnosis, risk stratification, and management. Am J Hematol 2011; 86: 928-48.

5. Wright A, Wijeratne A, Hung T, et al. Prevalence and severity of pruritus and quality of life in patients with cutaneous T-cell lymphoma. J Pain Symptom Manage 2013; 45: 114-9.

6. Vij A, Duvic M, Prevalence and severity of pruritus in cutaneous T cell lymphoma. Int I Dermatol 2012; 51: 930-4.

7. Sonkoly E, Muller A, Lauerma Al, et al. IL-31: a new link between $T$ cells and pruritus in atopic skin inflammation. J Allergy Clin Immunol 2006; 117: 411-7.

8. Raap U, Wichmann K, Bruder M, et al. Correlation of IL-31 serum levels with severity of atopic dermatitis. J Allergy Clin Immunol 2008; 122: 421-3.

9. Zhang Q, Putheti P, Zhou Q, et al. Structures and biological functions of IL-31 and IL-31 receptors. Cytokine Growth Factor Rev 2008; 19: 348-56.

10. Hermanns HM. Oncostatin M and interleukin-31: cytokines, receptors, signal transduction and physiology. Cytokine Growth Factor Rev 2015; 26: 545-58.

11. Cornelissen C, Lüscher-Firzlaff J, Baron JM, et al. Signaling by IL-31 and functional consequences. Eur J Cell Biol 2012; 91: 552-66.

12. Bilsborough J, Leung DY, Maurer M, et al. IL-31 is associated with cutaneous lymphocyte antigen-positive skin homing $\mathrm{T}$ cells in patients with atopic dermatitis. J Allergy Clin Immunol 2006; 117: 418-25.

13. Dillon SR, Sprecher C, Hammond A, et al. Interleukin 31, a cytokine produced by activated T cells, induces dermatitis in mice. Nat Immunol 2004; 5: 752-60.

14. Cevikbas F, Wang X, Akiyama T, et al A sensory neuronexpressed IL-31 receptor mediates T helper cell-dependent itch: involvement of TRPV1 and TRPA1. J Allergy Clin Immunol 2014; 133: 448-60.

15. Miyagaki T, Sugaya M. Erythrodermic cutaneous T-cell lymphoma: how to differentiate this rare disease from atopic dermatitis. J Dermatol Sci 2011; 64: 1-6.
16. Suga, H, Sugaya M, Miyagaki, T, et al. Skin barrier dysfunction and low antimicrobial peptide expression in cutaneous T-cell lymphoma. Clinl Cancer Res 2014; 20: 4339-48.

17. Papadavid E, Economidou J, Psarra A, et al. The relevance of peripheral blood T-helper 1 and 2 cytokine pattern in the evaluation of patients with mycosis fungoides and Sezary syndrome. Br J Dermatol 2003; 148: 709-18.

18. Saleem MD, Oussedik E, D’Amber V, et al. Interleukin-31 pathway and its role in atopic dermatitis: a systematic review. J Dermatol Treat 2017; 28: 591-9.

19. Gangemi S, Quartuccio S, Casciaro M, et al. Interleukin 31 and skin diseases: a systematic review. Allergy Asthma Proc 2017; 38: 401-8.

20. Moher D, Liberati A, Tetzlaff J, et al. Preferred reporting items for systematic reviews and meta-analyses: the PRISMA statement, J Clin Epidemiol 2009; 62: 1006-12.

21. Namer B, Carr R, Johanek LM, et al. Separate peripheral pathways for pruritus in man. J Neurophysiol 2008; 100: 2062-9.

22. Hassan I, Haji MI. Understanding itch: an update on mediators and mechanisms of pruritus. Indian J Dermatol Venereol Leprol 2014; 80: 106-14.

23. Yosipovitch G, Rosen JD, Hashimoto T. Itch: from mechanism to (novel) therapeutic approaches. J Allergy Clin Immunol 2018; 142: 1375-90.

24. Paus R, Schmelz M, Biro T, et al. Frontiers in pruritus research: scratching the brain for more effective itch therapy. J Clin Invest 2006; 116: 1174-86.

25. Tey HL, Yosipovitch G. Targeted treatment of pruritus: a look into the future. Br J Dermatol 2011; 165: 5-17.

26. Grimstad O, Sawanobori Y, Vestergaard C, et al. Anti-interleukin-31-antibodies ameliorate scratching behaviour in NC/ Nga mice: a model of atopic dermatitis. Exp Dermatol 2009; 18: 35-43.

27. Arai I, Tsuji M, Miyagawa K, et al. Repeated administration of IL-31 upregulates IL-31 receptor A (IL-31RA) in dorsal root ganglia and causes severe itch-associated scratching behaviour in mice. Exp Dermatol 2015; 24: 75-8.

28. Kasutani K, Fujii E, Ohyama S, et al. Anti-IL-31 receptor antibody is shown to be a potential therapeutic option for treating itch and dermatitis in mice. Br J Pharmacol 2014; 171: 5049-58.

29. Oyama S, Kitamura H, Kuramochi T, et al. Cynomolgus monkey model of interleukin-31-induced scratching depicts blockade of human interleukin-31 receptor A by a humanized monoclonal antibody. Exp Dermatol 2018; 27: 14-21.

30. Hawro T, Saluja R, Weller K, et al. Interleukin-31 does not induce immediate itch in atopic dermatitis patients and healthy controls after skin challenge. Allergy 2014; 69: 113-7.

31. Meng J, Moriyama M, Feld M, et al. New mechanism underlying IL-31-induced atopic dermatitis. J Allergy Clin Immunol 2018; 141: 1677-89.

32. Bağci IS, Ruzicka T. IL-31: a new key player in dermatology and beyond. J Allergy Clin Immunol 2018; 141: 858-66.

33. Ferretti E, Tripodo C, Pagnan G, et al. The interleukin (IL)-31/ IL-31R axis contributes to tumor growth in human follicular lymphoma. Leukemia 2015; 29: 958-67.

34. Storan ER, O'Gorman SM, McDonald ID, et al. Role of cytokines and chemokines in itch. Handb Exp Pharmacol 2015; 226: 163-76.

35. Nemoto O, Furue M, Nakagawa H. The first trial of CIM331, a humanized antihuman interleukin-31 receptor A 52. antibody, in healthy volunteers and patients with atopic dermatitis to evaluate safety, tolerability and pharmacokinetics of a single dose in a randomized, double-blind, placebo-controlled study. Br I Dermatol 2016; 174: 296-304. 
36. Ruzicka T, Hanifin JM, Furue M, et al. Anti-interleukin-31 receptor A antibody for atopic dermatitis. N Engl J Med 2017; 376: 826-35.

37. Kim S, Kim HJ, Yang HS, et al. IL-31 serum protein and tissue mRNA levels in patients with atopic dermatitis. Ann Dermatol 2011; 23: 468-73.

38. Sokołowska-Wojdyło M, Gleń J, Zabłotna M, et al. The frequencies of haplotypes defined by three polymorphisms of the IL-31 gene: $-1066,-2057$, and IVS2+ 12 in Polish patients with atopic dermatitis. Int J Dermatol 2015; 54: 62-7.

39. Neis MM, Peters B, Dreuw A, et al. Enhanced expression levels of IL-31 correlate with IL-4 and IL-13 in atopic and allergic contact dermatitis. J Allergy Clin Immunol 2006; 118: 930-7.

40. Nobbe S, Dziunycz P, Mühleisen B, et al. IL-31 expression by inflammatory cells is preferentially elevated in atopic dermatitis. Acta Derm Venereol 2012; 92: 24-8.

41. Hong $\mathrm{CH}, \mathrm{Yu} \mathrm{HS}$, Ko YC, et al. Functional regulation of interleukin-31 production by its genetic polymorphism in patients with extrinsic atopic dermatitis. Acta Derm Venereol 2012; 92: 430-2.

42. Lan CCE, Tu HP, Wu CS, et al. Distinct SPINK5 and IL-31 polymorphisms are associated with atopic eczema and nonatopic hand dermatitis in Taiwanese nursing population. Exp Dermatol 2011; 20: 975-9.

43. Raap U, Weißmantel S, Gehring M, et al. IL-31 significantly correlates with disease activity and Th2 cytokine levels in children with atopic dermatitis. Pediatr Allergy Immunol 2012; 23: 285-8.

44. Ezzat MHM, Hasan ZE, Shaheen KYA. Serum measurement of interleukin-31 (IL-31) in paediatric atopic dermatitis: elevated levels correlate with severity scoring. J Eur Acad Dermatol Venereol 2011; 25: 334-9.

45. Siniewicz-Luzeńczyk K, Stańczyk-Przyłuska A, Zeman K. Correlation between serum interleukin-31 level and the severity of disease in children with atopic dermatitis. Adv Dermatol Allergol 2013; 30: 282-5.

46. Szegedi K, Kremer AE, Kezic S, et al. Increased frequencies of IL-31-producing T cells are found in chronic atopic dermatitis skin. Exp Dermatol 2012; 21: 431-6.

47. Kato A, Fujii E, Watanabe T, et al. Distribution of IL-31 and its receptor expressing cells in skin of atopic dermatitis. J Dermatol Sci 2015; 74: 229-35.

48. Hamid Q, Boguniewicz M, Leung DYM. Differential in situ cytokine gene expression in acute versus chronic atopic dermatitis. J Clin Invest 1994; 94: 870-6.

49. Horejs-Hoeck J, Schwarz H, Lamprecht S, et al. Dendritic cells activated by IFN-gamma/STAT1 express IL-31 receptor and release proinflammatory mediators upon IL-31 treatment. J Immunol 2012; 188: 5319-26.

50. Nattkemper LA, Martinez-Escala ME, Gelman AB, et al. Cutaneous T-cell lymphoma and pruritus: the expresion of IL-31 and its receptors in the skin. Acta Derm Veneteol 2016; 96: 894-8.

51. Malek M, Gleń J, Rębała K, et al. IL-31 does no correlate to pruritus related to early stage cutaneous T-cell lymphomas but is involved in pathogenesis of the disease Acta Derm Venereol 2015; 95: 283-8.

52. Singer EM, Shin DB, Nattkemper LA, et al. IL-31 is produced by the malignant T-cell population in cutaneous T-cell lymphoma and correlates with CTCL pruritus. J Invest Dermatol 2013; 133: 2783-5.

53. Ohmatsu H, Sugaya M, Suga H, et al. Serum IL-31 levels are increased in patients with cutaneous T-cell lymphoma. Acta Derm Venereol 2012; 92: 282-3.
54. Mobs M, Gryzik S, Haidar A, et al. Analysis of the IL-31 pathway in Mycosis fungoides and Sezary syndrome. Arch Dermatol Res 2015; 307: 479-85. 